\title{
Thermal and thermo-catalytic degradation of polyolefins as a simple and efficient method of landfill clearing
}

\author{
Karina Tomaszewska, Joanna Kałużna-Czaplińska, Wojciech K. Jóźwiak \\ Technical University of Lodz, Institute of General and Ecological Chemistry, ul. Żeromskiego 116, 90-924 Łódź, Poland, \\ e-mail:karinatom@poczta.onet.pl
}

\begin{abstract}
Thermal degradation of the low density polyethylene (LDPE), polypropylene (PP) and the municipal waste plastics was investigated. The thermo-catalytic degradation of LDPE and PP was studied in the presence of the following catalysts: four different types of montmorillonite: K5, K10, K20, K30 and - for comparison - zeolites (natural - clinoptilolite, $\mathrm{Y}_{\mathrm{Na}+}$ and $\mathrm{Y}_{\mathrm{H}+}$ ). Thermal analyses TG-DTA-MS of polymers and polymercatalyst mixtures were carried out in an argon flow atmosphere in isothermal and dynamic conditions. The following order was found: in lowering the reaction temperature for LDPE degradation $Y_{\mathrm{H}+}>\mathrm{mK} 5>\mathrm{mK} 20$ $=\mathrm{mK} 30>\mathrm{mK} 10>\mathrm{NZ}>\mathrm{Y}_{\mathrm{Na}+}$; for $\mathrm{PP}$ degradation: $\mathrm{mK} 20>\mathrm{mK} 5=\mathrm{mK} 30>\mathrm{mK} 10>\mathrm{Y}_{\mathrm{H}+}>\mathrm{NZ}>$ $\mathrm{Y}_{\mathrm{Na}}$. The activity tests were carried out in a stainless steel batch reactor under atmospheric pressure in a wide temperature range of up to $410^{\circ} \mathrm{C}$, and using the atmosphere of argon flow. The liquid products were analysed by the GC-MS method. The hydrocarbons in the liquid products from thermal degradation of polymers were broadly distributed in the carbon fractions of $\mathrm{C}_{8}$ to $\mathrm{C}_{26}$ - for LDPE and $\mathrm{C}_{6}$ to $\mathrm{C}_{31}$ for PP.
\end{abstract}

Keywords: Polyethylene, polypropylene, polyolefins, municipal waste plastics, thermal degradation, thermocatalytic degradation, liquid fuels component, kinetics.

\section{INTRODUCTION}

Plastics are applied in almost every sector of the world economy, including building, engineering, the automotive industry, electronic and electrical engineering, medicine, laboratory and agriculture. However, the most popular application of plastics is in the production of various types of packaging ${ }^{1}$. Due to the advantages of polymers, such as durability, low price and thermal, chemical and microbiological resistance, plastics (especially polyolefins: polyethylene - PE and polypropylene - PP) have effectively replaced traditional materials like paper, glass and wood. This is beneficial from an economic point of view (plastics are usually much cheaper than traditional packaging) but not from an ecological one, since all the advantages of plastics which contribute to their large share in the packaging market become major disadvantages when they are transformed into waste. Material or chemical recycling of this type of waste is often unprofitable and their large volume together with the relatively low weight of the used plastics leads to the rapid expansion of landfill sites. This is a real environmental problem ${ }^{2,3}$.

The increasing amount of waste stored in landfills, accompanied by the significant depletion of coal, natural gas and crude oil reserves are the motivation for our work on an efficient method for the neutralization of polyolefin wastes and production of fuels in thermal cracking processes, in which the plastics recovered from wastes are thermally or thermo-catalytically degraded into valuable oils. There were two major aims of this work:

1. to find kinetic data, such as energy activation, of thermal degradation of polyolefins;

2. to find a correlation between the kind of the applied catalyst and their thermo-catalytic activity.

\section{EXPERIMENTAL}

\author{
Materials \\ In the present work the thermal degradation processes \\ of: \\ - low density polyethylene pellets - LDPE (Aldrich: \\ density $0.925 \mathrm{~g} \cdot \mathrm{cm}^{-3}$ ) \\ - polypropylene pellets - PP (Aldrich: isotactic, den- \\ sity $0.900 \mathrm{~g} \cdot \mathrm{cm}^{-3}$ ), \\ - municipal waste plastics: PE + PP (kindly delivered \\ by the company Posiadalo) \\ were investigated. In the thermo-catalytic degradation \\ processes of LDPE and PP the following catalysts were \\ used: four different kinds of montmorillonite (Fluka): \\ $\mathrm{K} 5, \mathrm{~K} 10, \mathrm{~K} 20, \mathrm{~K} 30$ denoted as: $\mathrm{mK} 5, \mathrm{mK} 10, \mathrm{mK} 20$, \\ mK30; zeolites: natural - clinoptilolite (Erem, Poland) \\ and $\mathrm{Y}$ type (IZS Matwy, Aldrich) described respectively \\ as NZ, $\mathrm{Y}_{\mathrm{Na}+}$ and $\mathrm{Y}_{\mathrm{H}+}$. The physical properties of these \\ catalysts were presented in our previous work ${ }^{4}$.
}

\section{Methods}

Thermal analyses TG-DTA-MS were carried out in an argon flow (volume velocity $40 \mathrm{~cm}^{3} \cdot \mathrm{min}^{-1}$ ) with the use of a Setaram thermobalance unit (Setsys 16/18) connected to the Balzers quadrupole mass spectrometer (Thermostar). The weight of each sample was about 16 mg. Two types of experiments: isothermal and dynamic were performed. In the first type, the polymer sample was heated at $20^{\circ} \mathrm{C} \cdot \mathrm{min}^{-1}$ to $300^{\circ} \mathrm{C}$. After 5 minutes it was heated again at $20^{\circ} \mathrm{C} \cdot \mathrm{min}^{-1}$ to the required temperature (ranged from 380 to $480^{\circ} \mathrm{C}$ ). In the second type, the polymer was mixed with the catalyst and subjected to different heating rates of 5,10 and $20^{\circ} \mathrm{C} \cdot \mathrm{min}^{-1}$ up to $700^{\circ} \mathrm{C}$. The thermo-catalytic degradation of the polymer was performed using a mass ratio polymer/catalyst $=10 / 1$.

The activity tests were carried out in an in-house designed stainless steel reactor by batch operation under atmospheric pressure. The sample (50 $\mathrm{g}$ of polymer - for 
the thermal degradation or the same sample mass with $5 \mathrm{~g}$ of catalyst - for the catalytic degradation) was loaded into the reactor. The air of the reactor was purged with an argon flow. The reactor was heated to the reaction temperature at a rate of $5^{\circ} \mathrm{C} \cdot \mathrm{min}^{-1}$ and held until no more liquid was produced. The outlet of the reactor was connected to a condenser, maintained at $15^{\circ} \mathrm{C}$.

The polymer degradation reaction products were: gases, liquids and solid residues. The yield of the liquid product was determined by the amount of liquid collected in a receiver. The yield of solid residues was estimated by the mass of waxy compounds or carbon deposit and catalyst inside the reactor after the experiment. The yield of gas products was calculated as the difference between the amounts of polymer plus catalyst and the yield of liquids and residues. The desired products - liquids - were detected during the GC or GC-MS analyses. The distribution of the obtained hydrocarbons in liquid products was analysed by an Agilent Technologies 6890N Network GC coupled to 5973 Network Mass Selective Detector.

\section{RESULTS AND DISCUSSION}

Isothermal TG-DTA-MS analyses of LDPE and PP thermal degradation were carried out at a constant temperature, ranged from 380 to $480^{\circ} \mathrm{C}$, to determine the effect of temperature on the duration and the activation energy of the reaction. The results of LDPE thermal degradation at different temperatures are presented in Figure $1 \mathrm{a}$, whereas the results obtained for thermal degradation of pure PP pellets are presented in Figure $1 \mathrm{~b}$.

In Figure 1. one can observe that the time necessary to complete the degradation of LDPE and PP in isothermal processes is related to the temperature of the degradation process. At the lowest temperature $-380^{\circ} \mathrm{C}$, the LDPE degradation reaction takes over 1000 minutes, while at

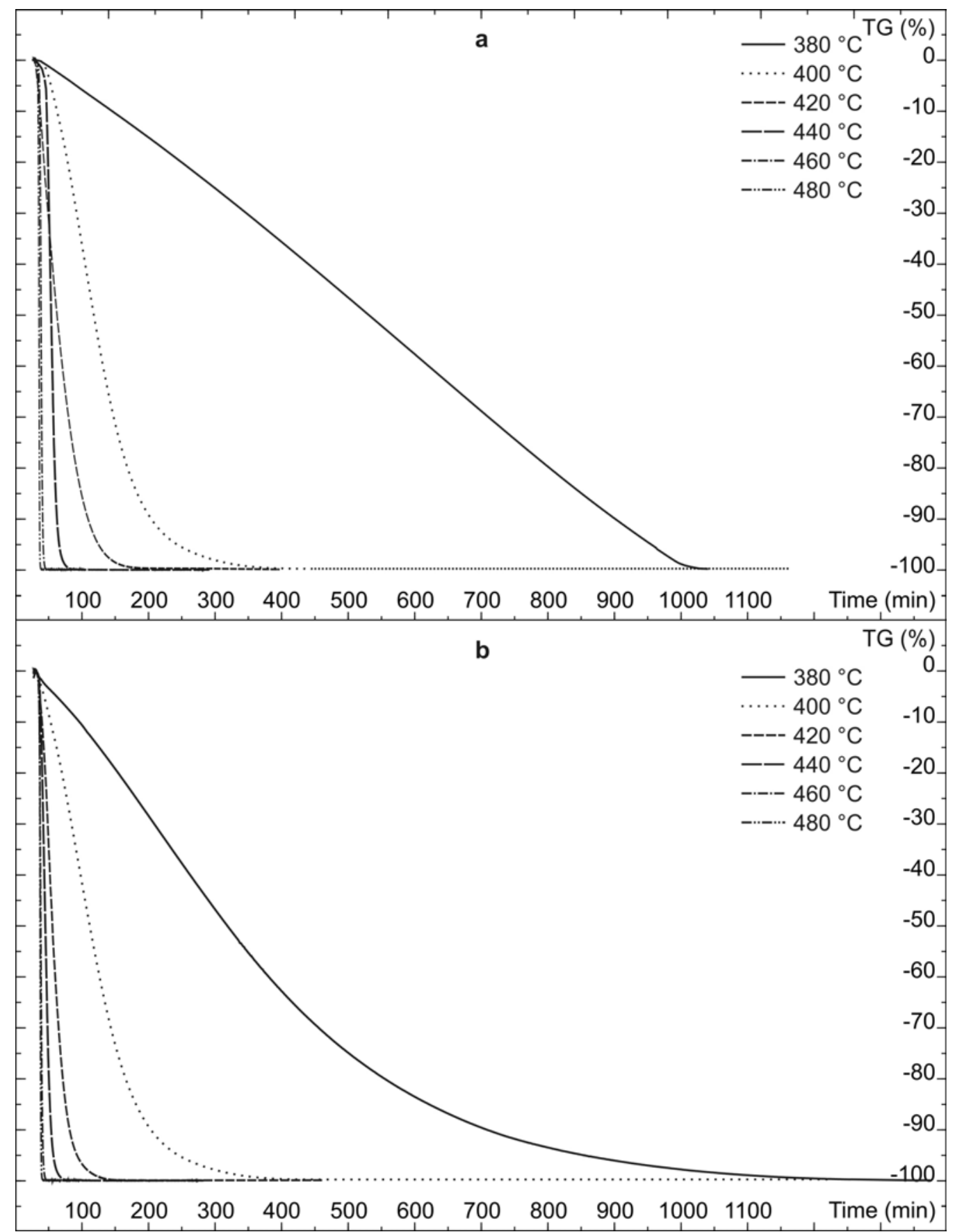

Figure 1. The kinetic curves of polymer mass loss in function of time reaction $\Delta \mathrm{m}=\mathrm{f}(\mathrm{t})$ for different temperature of a - LDPE, b - PP degradation in the range $380-480^{\circ} \mathrm{C}$ 
$480^{\circ} \mathrm{C}$ only 12 minutes. Also, the PP degradation process carried out at $380^{\circ} \mathrm{C}$ takes over 1300 minutes, while at $480^{\circ} \mathrm{C}$ only 13 minutes.

The results of the isothermal TG-DTA-MS analyses of LDPE and PP thermal degradation were also used to estimate the activation energy of the reaction. To determine the activation energy the Arrhenius equation was used $^{5-7}$ :

$\mathrm{k}=\mathrm{A} \cdot \mathrm{e}^{\frac{-\mathrm{E}_{\mathrm{a}}}{\mathrm{R} \cdot \mathrm{T}}}$

where:

$\mathrm{k}$ - reaction rate constant;

A - pre-exponential factor $\left(\mathrm{s}^{-1}\right)$;

$\mathrm{E}_{\mathrm{a}}$ - activation energy $\left(\mathrm{J} \cdot \mathrm{mol}^{-1}\right)$;

$\mathrm{R}$ - gas constant $\left(8.314 \mathrm{~J} \cdot \mathrm{mol}^{-1} \cdot \mathrm{K}^{-1}\right)$;

$\mathrm{T}$ - temperature $(\mathrm{K})$.

For the thermal LDPE and PP degradation, the reaction rate constant was determined from the following equation:

$\mathrm{k}=\frac{\mathrm{d} \alpha}{\mathrm{dt}}$

where:

$\alpha$ - conversion degree of polymer degradation;

$\mathrm{t}$ - time of polymer degradation.

On the basis of constant $\mathrm{k}$ the rate graph of the function of temperature:

$\ln \mathrm{k}=\mathrm{f}\left(\frac{1}{\mathrm{~T}}\right)$ was drawn. The respective graphs of the function described by equation 3. are presented for LDPE (Fig. 2.) and PP (Fig. 3.).

For LDPE and PP degradation this relation is a linear function, therefore the Arrhenius equation in a logarithmic form:

$\ln \mathrm{k}=\frac{-\mathrm{E}_{\mathrm{a}}}{\mathrm{R}} \cdot \frac{1}{\mathrm{~T}}+\ln \mathrm{A}$

is fulfilled. This confirms the assumption that in the considered temperature range the activation energy and the pre-exponential factor have a constant value. The linear regression method was used to determine the coefficients $\mathrm{a}$ and $\mathrm{b}$ of a linear function:

$\ln \mathrm{k}=\mathrm{a} \cdot \frac{1}{\mathrm{~T}}+\mathrm{b}$

and then, on their basis - the activation energy and the pre-exponential factor were determined:

for LDPE thermal degradation:

$\mathrm{E}_{\mathrm{a}} \approx 180 \mathrm{~kJ} \cdot \mathrm{mol}^{-1}$

$\mathrm{A}=7.95 \cdot 10^{10} \mathrm{~s}^{-1}$

for PP thermal degradation:

$\mathrm{E}_{\mathrm{a}} \approx 190 \mathrm{~kJ} \cdot \mathrm{mol}^{-1}$

$\mathrm{A}=4.12 \cdot 10^{11} \mathrm{~s}^{-1}$

Both values of the activation energy $E_{a}$ and the preexponential factor A strongly confirm the analogical mechanism of the low density polyethylene and polypropylene thermal degradation.

The thermal degradation of polyethylene and polypropylene was also studied in dynamic conditions

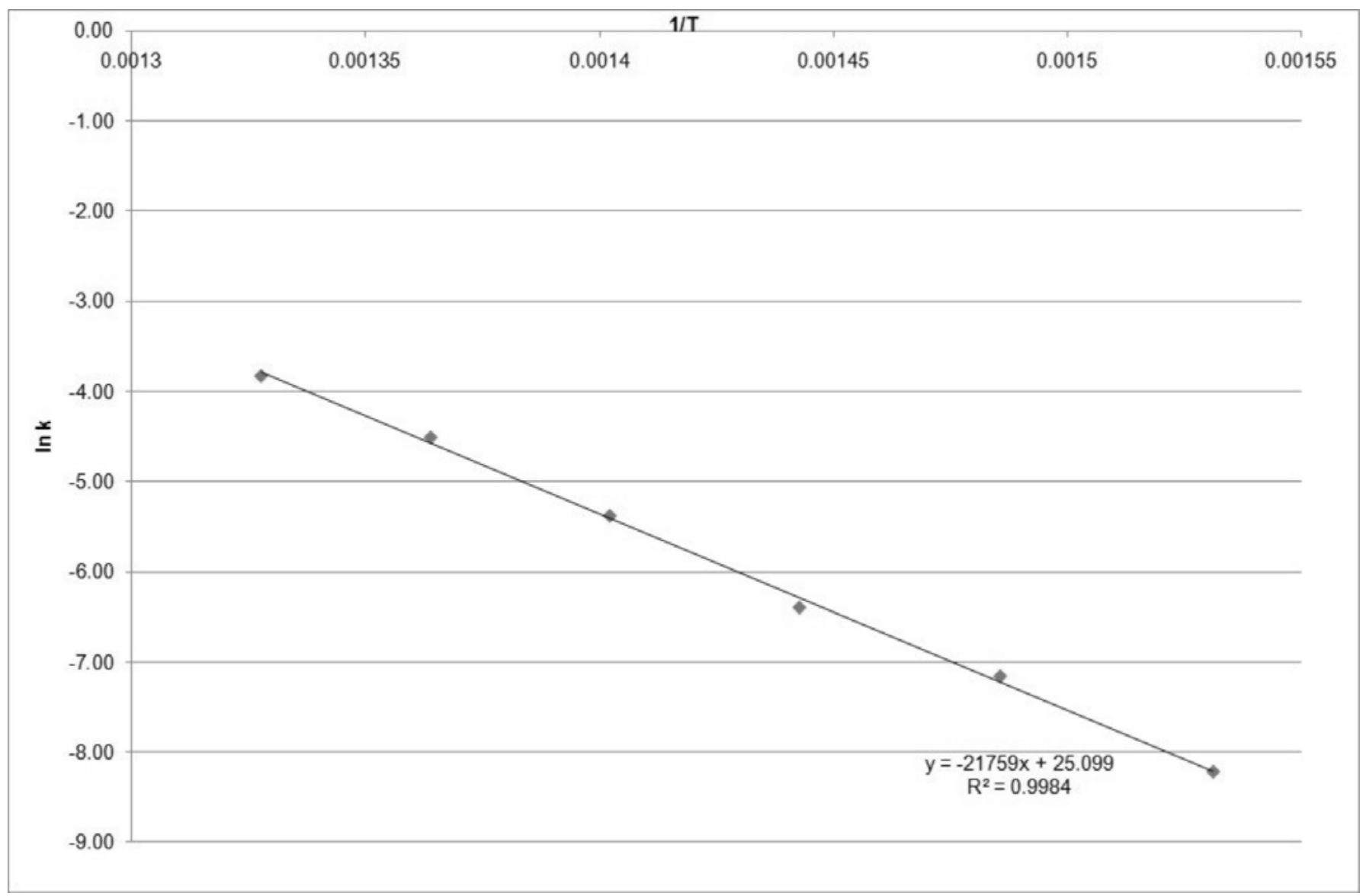

Figure 2. The graph of the function $\ln k=f\left(\frac{1}{T}\right)$ for thermal degradation of LDPE 


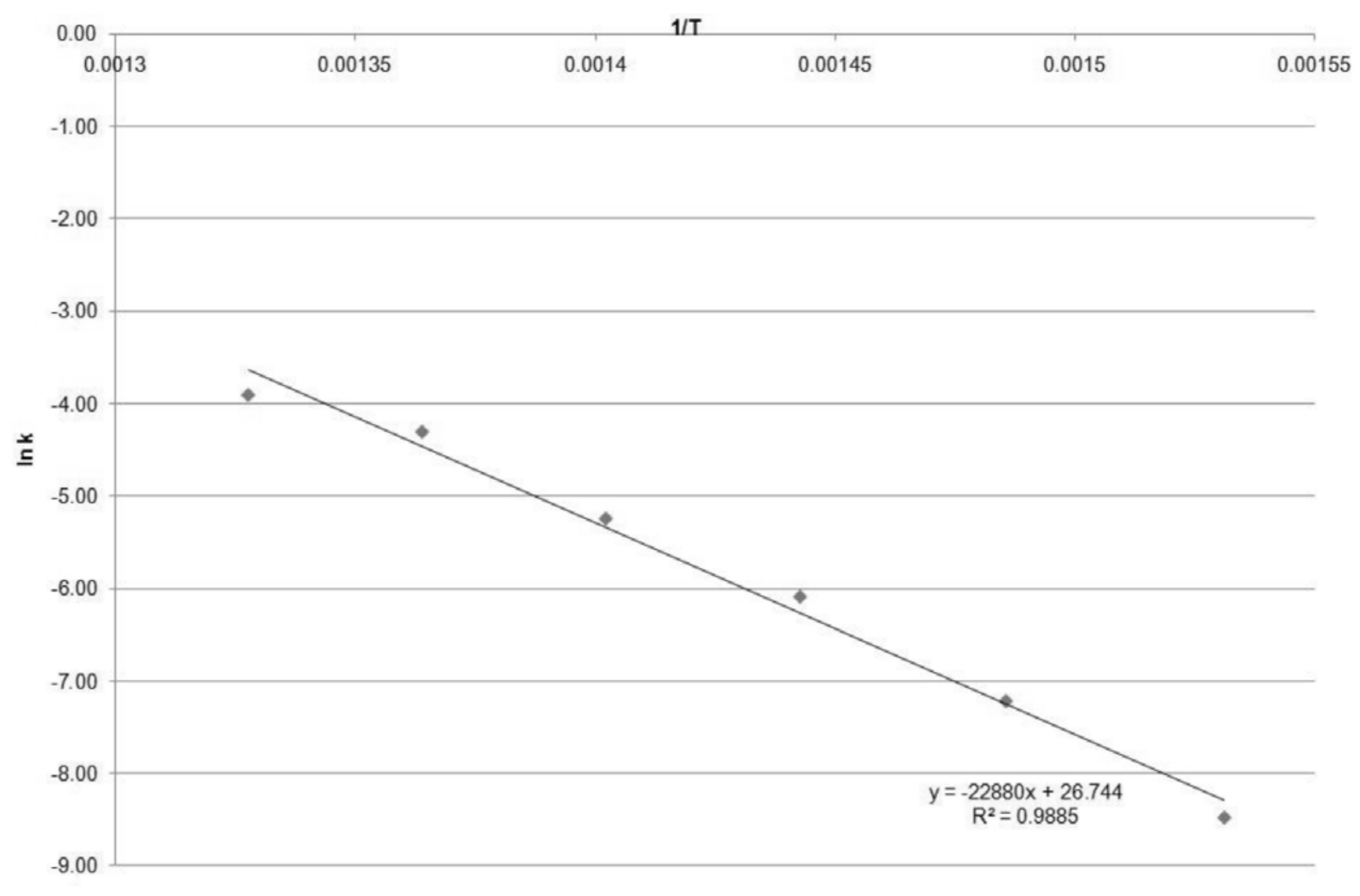

Figure 3. The graph of the function $\ln k=f\left(\frac{1}{T}\right)$ for thermal degradation of PP

with different heating rates, to determine the influence of the heating rate and kind of catalyst used, on the reaction temperature. The results TG-DTA obtained for LDPE and PP degradation were compared with the curves obtained during the thermal degradation of municipal waste plastics, too (Fig. 4.).

The TG curve of the thermal degradation of LDPE (Fig. 4a.) indicates that this process occurs in the temperature range $370-510^{\circ} \mathrm{C}$. It is accompanied by mass loss equal to about $100 \%$. This is a one-step endothermic process. One can also observe a smaller endothermic effect at the temperature of $113^{\circ} \mathrm{C}$, corresponding to the melting process of the polymer.

Polypropylene thermal degradation takes place in the temperature range from 345 to $493^{\circ} \mathrm{C}$ and melting of the sample occurs at the temperature of $167^{\circ} \mathrm{C}$ (Fig. 4b.). The thermal PP degradation process is endothermic and completed (100\% mass loss on the TG curve), but this time it is not a one-step reaction. It runs in a series of several strongly overlapping steps.

The TG-DTA curves of the thermal degradation of municipal waste plastics presented in Figure 4c, confirm that it is mainly a mixture of polyethylene and polypropylene with relatively small amounts of impurities in the form of dust and sand (about $5 \%$ ). The temperature range of the decomposition process is $370-510^{\circ} \mathrm{C}$ and melting point is about $130^{\circ} \mathrm{C}$, which suggest that a significant part of this waste is polyethylene. Whereas the incomplete separation of the endothermic peaks on the DTA curve in the range of degradation temperature, confirms the presence of polypropylene in the tested material.
The TG curves for the degradation of both plastics with different heating rates $\left(5,10,20^{\circ} \mathrm{C} \cdot \mathrm{min}^{-1}\right)$ indicate that the lower value of this parameter decreases the initial temperature of polymer decomposition insignificantly.

The TG-DTA results obtained for the thermal degradation of pure individual polymers: LDPE and PP (without catalyst) and those mixed with catalyst (mK5, mK10, $\mathrm{mK} 20, \mathrm{mK} 30, \mathrm{NZ}, \mathrm{Y}_{\mathrm{Na}+}$ or $\mathrm{Y}_{\mathrm{H}+}$.) in an argon flow at $10^{\circ} \mathrm{C} \cdot \mathrm{min}^{-1}$ are shown in Figure 5 . The polymer mass presented in this figure is reduced by the mass of the added catalyst, which does not change during the experiment.

The application of any catalyst in the LDPE degradation decreases not only the time of the process but also the reaction temperature (Fig. 5a.). The thermal degradation at a ramp rate of $10^{\circ} \mathrm{C} \cdot \mathrm{min}^{-1}$ takes place in the temperature range $390-510^{\circ} \mathrm{C}$, while the catalytic polymer decompositions on the K-type montmorillonite and $\mathrm{Y}_{\mathrm{H}+}$ catalysts begin below $300^{\circ} \mathrm{C}$ (even at $250^{\circ} \mathrm{C}$ for $\mathrm{mK} 5$ and $\mathrm{Y}_{\mathrm{H}+}$ catalysts). In the case of the PP thermo-catalytic degradation process, the use of any catalyst causes the lowering of the temperature of this reaction from 345 $493^{\circ} \mathrm{C}$ to even $210-435^{\circ} \mathrm{C}$ for mK20 catalyst. The acceleration of thermal degradation of LDPE and PP over montmorillonite $\mathrm{K}$-type and $\mathrm{Y}_{\mathrm{H}+}$ zeolite of this study can be explained by the presence of the active acid sites on the catalyst surface and large pore volume. A large pore volume allows the penetration of the polymer's macromolecules into the catalyst, where further degradation of polymer chains takes place.

The GC-MS analyses of the liquid products of LDPE and PP thermal degradation were conducted to determine 


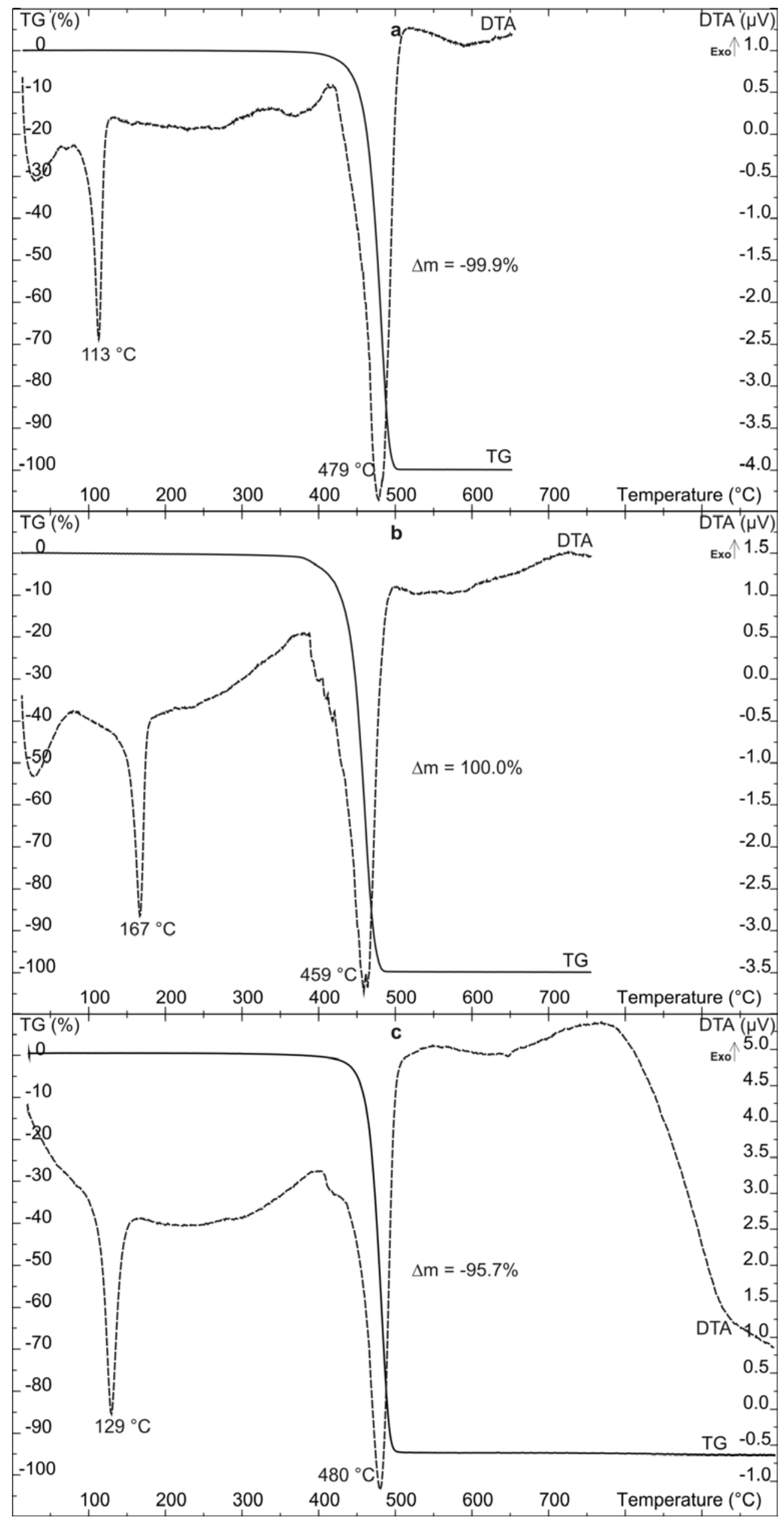

Figure 4. TG-DTA curves of thermal degradation of: a - LDPE, b - PP, c - municipal waste plastics in an argon flow at the heating rate of $10^{\circ} \mathrm{C} \cdot \mathrm{min}^{-1}$ 


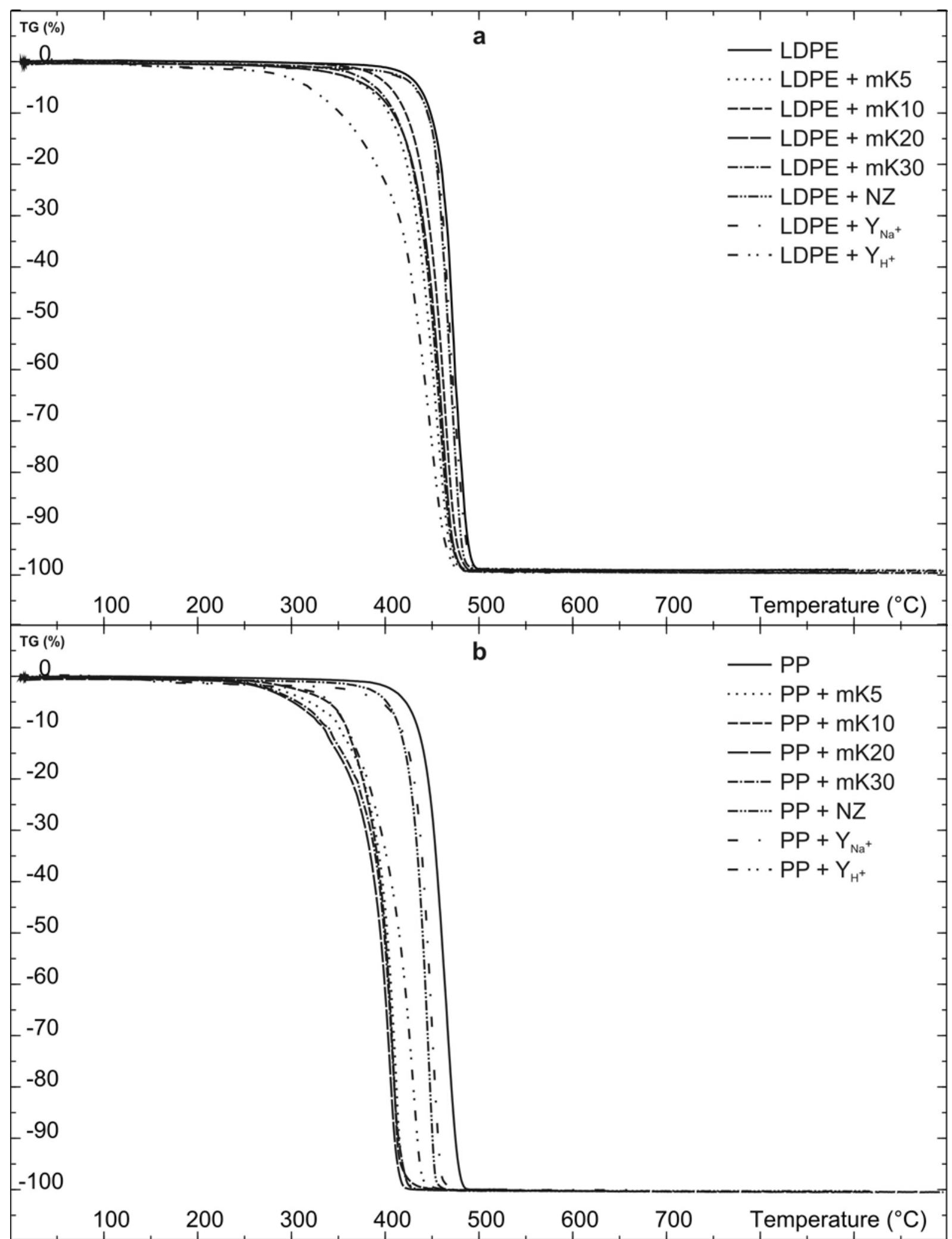

Figure 5. The TG curves of the polymer mass loss during: a - LDPE, b - PP thermal and thermo-catalytic degradation processes in an argon flow over different catalysts at a heating rate of $10^{\circ} \mathrm{C} \cdot \mathrm{min}^{-1}$

the composition of the hydrocarbon mixture. In Fig. 6 the carbon number distribution of hydrocarbons in liquid products obtained by thermal degradation of LDPE is compared with that obtained by thermal degradation of PP. Table 1. also shows the composition of the liquid products.

In the thermal degradation of LDPE and PP, many hydrocarbons with a wide-ranging distribution of carbon atom numbers $\left(\mathrm{C}_{8}-\mathrm{C}_{26}\right.$ for LDPE and $\mathrm{C}_{6}-\mathrm{C}_{31}$ for PP) are formed (Fig. 6). The liquid products of both LDPE and PP decomposition processes (Table 1.) are mainly a mixture of alkanes, alkenes, cycloalkanes and cycloalkenes without the compounds containing heteroatoms like $\mathrm{Cl}, \mathrm{N}$ or S. One can observe only the compounds containing oxygen in the molecules. This may have been caused by the incomplete removal of air from the polymer pellets before starting the degradation processes. The application of any catalyst in these types of reaction leads to the 


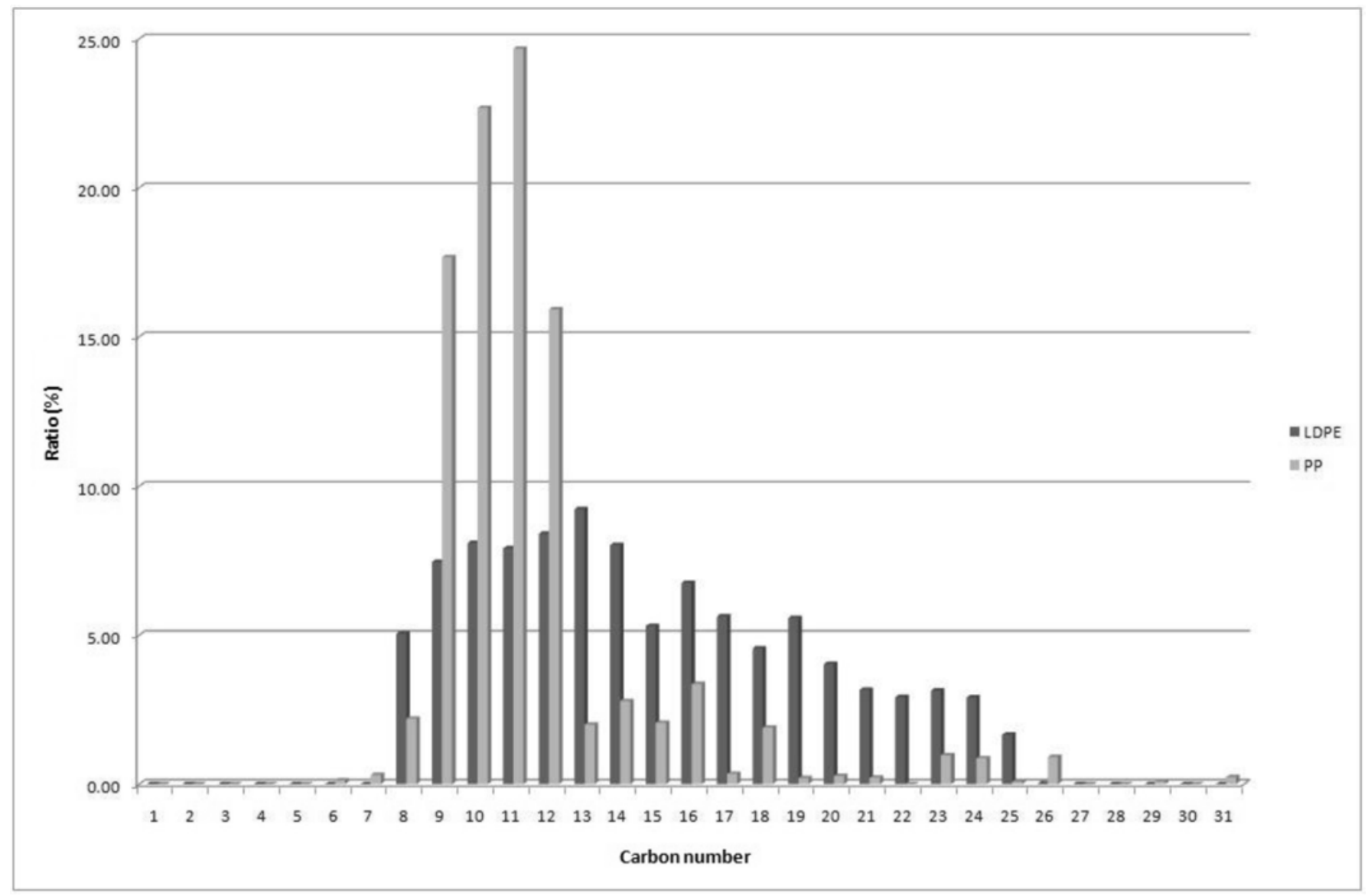

Figure 6. The distribution of hydrocarbons in the liquid products formed in the LDPE and PP thermal degradation at $410^{\circ} \mathrm{C}$ as a function of carbon number

Table 1. The composition of the liquid products [wt \%] in thermal degradation of LDPE and PP at $410{ }^{\circ} \mathrm{C}$

\begin{tabular}{|c|c|c|c|c|c|c|c|c|c|c|}
\hline & \multicolumn{4}{|c|}{ Percent of total - hydrocarbons } & \multicolumn{3}{c|}{ Percent of total - compounds with: } \\
\cline { 2 - 12 } & Alkanes & Alkenes & Alkynes & Cykloalkanes & Cykloalkenes & Aromatics & Oxygen & Chlorine & Nitrogen & Sulphur \\
\hline LDPE & 36.49 & 41.46 & 0.55 & 12.78 & 8.23 & 0.21 & 0.16 & 0.00 & 0.00 & 0.00 \\
\hline PP & 15.74 & 26.06 & 0.47 & 51.74 & 2.13 & 2.65 & 1.13 & 0.00 & 0.00 & 0.00 \\
\hline
\end{tabular}

production of lighter hydrocarbons than in the non-catalytic processes.

\section{CONCLUSIONS}

- Selection of a suitable temperature of LDPE and PP degradation significantly decreases the reaction time (by even 100 times).

- Activation energy of thermal degradation of LDPE is equal to $180 \mathrm{~kJ} \cdot \mathrm{mol}^{-1}$ and active collision frequency factor (pre-exponential factor) equals $7.95 \cdot 10^{10} \mathrm{~s}^{-1}$.

- Activation energy of thermal degradation of PP is equal to $190 \mathrm{~kJ} \cdot \mathrm{mol}^{-1}$ and active collision frequency factor equals $4.12 \cdot 10^{11} \mathrm{~s}^{-1}$.

- The following order in lowering the temperature of polymer degradation was established:

- for LDPE: $\mathrm{Y}_{\mathrm{H}+}>\mathrm{mK} 5>\mathrm{mK} 20=\mathrm{mK} 30>\mathrm{mK} 10$ $>\mathrm{NZ}>\mathrm{Y}_{\mathrm{Na}+}$;

- for PP: $\mathrm{mK} 20>\mathrm{mK} 5=\mathrm{mK} 30>\mathrm{mK} 10>\mathrm{Y}_{\mathrm{H}+}>$ $\mathrm{NZ}>\mathrm{Y}_{\mathrm{Na}+}$.

- K-type montmorillonites greatly accelerate the degradation both of LDPE and PP and also decrease the temperature at which those processes occur.
- The hydrocarbons in the liquid products from thermal degradation of LDPE were broadly distributed in the carbon fractions of $\mathrm{C}_{8}$ to $\mathrm{C}_{26}$.

- The hydrocarbons in the liquid products from thermal degradation of PP were broadly distributed in the carbon fractions of $\mathrm{C}_{6}$ to $\mathrm{C}_{31}$, but hydrocarbons with carbon numbers from 9 to 12 dominate.

- The catalytic degradation products contain a relatively narrow distribution of hydrocarbons (lighter hydrocarbons).

\section{ACKNOWLEDGEMENT}

We would like to acknowledge Mr. Slawomir Posiadalo for his collaboration in this research.

The research reported in this paper is a part of a project conducted from 2007 to 2010 with the support of the Ministry of Science and Higher Education (grant no. N205 025 32/1352).

\section{LITERATURE CITED}

1. PlasticsEurope. (2009, October). The Compelling Facts About Plastics 2009. An analysis of European plastics production, demand and recovery for 2008. Retrieved Novem- 
ber 3. 2009, from http://www.plasticseurope.org/Documents/ Document/20100225141556-Brochure_UK_FactsFigures 2009_22sept_6_Final-20090930-001-EN-v1.pdf.

2. Rabek, J.F. (2008). Contemporary knowledge of polymers. Warszawa, Polska: Wydawnictwo Naukowe PWN (in Polish).

3. Mucha, M. (2002). Polymers and ecology. Łódź, Polska: Wydawnictwo Politechniki Łódzkiej (in Polish).

4. Tomaszewska, K. \& Jóźwiak, W.K. (2009). Aluminosilicates in thermo-catalytic degradation of low density polyethylene, Pol. J. Environ. Stud. 18 1B, 232 - 236.

5. Pielichowski, K. \& Njugana, J. (2005). Thermal degradation of polymeric materials. Shawbury, United Kingdom: Rapra Technology Limited.

6. Reverte, C., Dirion, J.L. \& Cabassud, M. (2007). Kinetic model identification and parameters estimation from TGA experiments. J. Anal. Appl. Pyrolysis 79, 297 - 305. DOI: 10.1016/j.jaap.2006.12.021.

7. Abate, L., Blanco, I., Pollicino, A. \& Recca, A. (2002). Determination of degradation apparent activation energy values of polymers Regression of kinetic parameters derived from TG data. J. Therm. Anal. Calorim. 70, 63 - 73. 